\title{
EFECTO LÁTIGO EN LA PLANEACIÓN DE LA CADENA DE ABASTECIMIENTO, MEDICIÓN Y CONTROL
}

\section{BULLWHIP EFFECT IN SUPPLY CHAIN, HOW TO MEASURE AND CONTROL}

\author{
Juan Carlos Mejía Villamizar \\ Ing. Industrial, Especialista en Gerencia en Logística Integral, \\ Auxiliar de Investigación Grupo PIT, Facultad de Ingeniería, \\ Universidad Militar Nueva Granada, Bogotá, Colombia, \\ juancarlosmejiav@gmail.com
}

Óscar Palacio León

Ing. Industrial, M.Sc, Doctorando en Ingeniería de la UC (Venezuela),

Doctorando en Proyectos de la UNINI (Mexico),

Profesor Asistente, Facultad de Ingeniería, Investigador Grupo PIT

Universidad Militar Nueva Granada, Bogotá, Colombia,

oscar.palacio@unimilitar.edu.co

Wilson Adarme Jaimes

Ing. Industrial, Ph.D., Profesor Asociado,

Facultad de Ingeniería, Investigador Grupo SEPRO.

Universidad Nacional de Colombia,

Sede Bogotá, Colombia,

wadarmej@unal.edu.co

Fecha de recepción: 12 de octubre de 2012

Fecha de aceptación: 22 de enero de 2014

\section{RESUMEN}

El Efecto Látigo (En idioma inglés Bullwhip Effect) es uno de los causantes de las fluctuaciones que experimenta la proyección de la demanda a medida que se aleja del mercado a lo largo de la Cadena de Suministro (CdS), como consecuencia de falta de coordinación y sincronización entre los agentes intervinientes (Proveedor, fabricante, distribuidor, mayorista, minorista). Esta situación afecta la planificación estratégica y operativa pretendida por las organizaciones que integran la CdS, en relación a los tiempos de respuesta, capacidad de almacenamiento, administración de los costos logísticos ocultos y procesos de negociación, entre otros aspectos. Una forma de atenuar este efecto, es que la CdS 
posea un sistema de información gerencial sincronizado bajo un ambiente cooperativo de negociación. Este escenario empresarial no siempre se suele dar fácilmente, motivo por el cual se propone un método de medición y control basado en técnicas de la Dinámica de Sistemas, específicamente, la aplicación de alisado exponencial y de Holt-Winters. Estos métodos permiten transmitir los mismos patrones de demanda recibidos, sin ningún tipo de variación a lo largo de la CdS.

Palabras clave: Efecto Látigo, Modelo EPQ, Agentes Logísticos, Parámetros de la Demanda, Nivel de Inventario.

\section{ABSTRACT}

The bullwhip effect, is one of the causes of the fluctuations experienced by the projection of demand as the market moves away along the supply chain (SC), as a result of lack of coordination and synchronization between the agents (supplier, manufacturer, distributor, wholesaler, retailer). This situation directly affects the strategic and operation al planning proposed by the member organizations the SC, in relation to response time, storage management, hidden logistics costs and negotiation processes, among others things. Undoubtedly, one way to mitigate this effect is that the SC has a system of management information synchronized under a collaborative environment for negotiation. This business scenario usually occurs does not always easy, reason why we propose a method of measurement and control based in techniques of System Dynamics specifically the application of exponential smoothing and Holt-Winters. These methods allow us to transmit the same demand patterns received, without any variation.

Keywords: Bullwhip Effect, EPQ Model, Agents Logistics, Demand Parameters, Inventory Level.

\section{INTRODUCCIÓN}

En una economía globalizada y dinámica como la actual, en donde los Tratados de Libre Comercio (TLC) están a la orden del portafolio gubernamental, se obligan a las firmas a reformular en el menor tiempo posible sus estrategias empresariales que les permitan de forma eficiente ajustarse a las nuevas condiciones impuestas por dichos TLC. En el mismo sentido, cada uno de los agentes logísticos intervinientes en una misma CdS deberán garantizar la satisfacción por el servicio prestado al cliente final, de modo que el flujo de dinero, información y materiales se den a través del desarrollo de las operaciones empresariales conjuntas que alimentan los inputs y outputs de la economía de un país [1]. Además, una CdS deberá estar integrada, sincronizada y coordinada (Es decir optimizada), para que los procesos conjuntos de mejora continua se vean reflejados en: i) Reducción en las necesidades de inventario; ii) Ahorros en 
costos de manutención, transporte y otros gastos de distribución; y iii) Disminución en los tiempos de respuesta [2].

La logística es la herramienta de la competitividad con la cual los actores miembros de una CdS, en particular; diseñan fórmulas empresariales, tales como: i) Mejorar el uso del cubo de almacenamiento; y ii) Negociar la Unidad de Carga Eficiente (UCE) de forma efectiva; con las cuales la CdS como un todo, busca racionalizar sus costos logísticos, situación que se ve obstaculizada por el denominado Efecto Látigo, como consecuencia de que este fenómeno administrativo transmite incertidumbre al interior de los procesos de gestión relacionados con el área de operaciones [2-3]. Las soluciones adoptadas por los agentes logísticos abarcan desde el uso intensivo de sistemas de información basados en Eventos Virtuales de Trabajo Colaborativo (EVTC) para el intercambio de datos sobre la demanda que experimentan los productos que administran, pasando por la centralización del control de los inventarios [4].

El presente artículo está dividido en cuatro apartados: El primero, realiza una descripción del Efecto Látigo. El segundo, describe las técnicas de planificación de la demanda sobre las cuales se cimenta el desarrollo matemático para la mitigación del Efecto Látigo al interior de la CdS, eje central del presente artículo. El tercero, desarrolla la experimentación del modelado matemático propuesto con datos de demanda provenientes de una Compañía Colombiana, líder en la línea de productos de blanqueadores a base de Hipoclorito de Sodio. En el último apartado se indican las conclusiones a las que llegó la presente investigación.

Se destaca que la solución propuesta por la investigación se basa en los lineamientos sugeridos por el doctor Ramón Julián Martín-Andino Benítez, en su Tesis Doctoral titulada "El efecto látigo (bullwhip) en las CdS y la dependencia de los agentes que las integran" [5], reconocido en el medio científico de la logística como experto en esta materia.

\section{MÉTODOS}

Las costumbres de los nacionales hacia el consumo progresivo de productos de limpieza y desinfección en lo corrido del Siglo XXI han modificado el mercado de oferentes en alguna forma, a tal punto, que compañías que manejaban este tipo de productos (Hipoclorito) como un residual de sus procesos productivos de línea, han venido fortaleciendo dichos procesos como respuesta a la búsqueda de abaratar sus precios finales en concordancia con sus operaciones logísticas [6-7]. Hay factores operativos, logísticos, financieros, comerciales, en los que se apoyan los tomadores de decisiones logísticas con respecto a los procesos de coordinación para viabilizar la existencia de los procesos de sincronización necesarios como garantes de una gestión administrativa conjunta en total armonía; la falencia de una sincronía en los sistemas de información por parte de los actores, así como él desinterés por una integración duradera por parte de los mismos, se constituyen en el caldo de cultivo propicio para 
la aparición del "Efecto Látigo". Los detonadores del Efecto Látigo se pueden condensar en las siguientes tres categorías [5]: i) Las debidas a la agrupación de órdenes de reposición motivadas por costos logísticos inalterables respecto a las unidades solicitadas; ii) Las derivadas de cambios en las topologías de las redes de suministro; y iii) Las que tienen su origen en perturbaciones de la demanda. La presente investigación se fundamenta en el estudio de las perturbaciones que experimenta la demanda, referidas a dos aspectos a saber: i) Las técnicas de pronóstico empleadas por las compañías para estimar sus necesidades de tesorería; y ii) Los cambios no planificados en el comportamiento de la demanda, motivados por deseos especulativos o por alcanzar niveles racionales de protección ante supuestas incertidumbres vía inventarios.

El proceso metodológico utilizado por los investigadores del presente estudio, se fundamentó en una revisión de la literatura científica que analizan el Efecto Látigo, sus métodos de medición y pautas aplicables; y a través del usos de la tecnología EVTC, en el área de planeación y logística de una compañía nacional líder en la manufacturación de productos con base química de hipoclorito de sodio; permitiendo tener una clara imagen de cómo son los procesos y lograr entender el impacto de los diferentes indicadores logísticos en dicha CdS. Con el objeto de poder describir de forma breve, las teorías y modelos que soportan la mitigación del bullwhip. El desempeño de las CdS de las industrias químicas está sujeto al grado de variabilidad que impone el distribuidor por sus preferencias de mercado, a modo de ejemplo si el blanqueador de ropa no es un elemento de primera necesidad para el consumidor, su intención de compra será influenciada por la disponibilidad efectiva del producto en góndola, independiente del plus que posea como tal dicho producto, (Aromas, combinaciones, promocionales) que el proveedor puede ofrecerle. Esta afirmación se deriva de la investigación iniciada a comienzos de 2010 por la Cámara de Sabores y Fragancias de la Asociación Nacional de Empresarios de Colombia (ANDI), la cual determinó que el volumen de las ventas globales a pesos constantes de hoy, en la línea de blanqueadores para ropa a nivel nacional, asciende a 72.537.890.000 COP [6-7].

El estudio se centralizó en los blanqueadores con base química de hipoclorito de sodio, producidos en el Departamento de Cundinamarca (Colombia). Empleando tecnología EVTC y observación directa in situ. Se tomó como lineamiento rector de orientación la Tesis Doctoral del Dr. Martín-Andino, en lo referente al método de estimación del bullwhip por medio de comparación de varianzas de la demanda, aplicando el modelo de gestión OUT (Order Up to Level) para gestión y reposición de pedidos. En relación a los datos obtenidos de la compañía, éstos no evidenciaron un componente estacional arraigado, motivo por el cual se empleó inicialmente, el modelo de alisado exponencial simple para estimar la demanda y en relación a la disponibilidad de datos, se utilizó como segundo modelo de alternancia a Holt-winters a criterio de los investigadores. Así mismo, se compararon los resultados obtenidos de la modelación 
de la demanda mensual para la firma, con miras a seleccionar de estos dos métodos el que mejor la estima.

\section{HISTORIA DEL EFECTO LÁTIGO}

En 1961 Jay Forrester, creador de la Dinámica de Sistemas, demostró la posibilidad de que la demanda cursada de un comprador a su proveedor incrementa su amplitud a medida que se remonta aguas arriba de la CdS [8], desde entonces han sido varias las hipótesis estudiadas que, aplicando diversas técnicas empíricas y matemáticas, describen como se origina este efecto en diversas CdS estudiadas a nivel orbital. Los estudios del fenómeno destacan varias líneas de actuación investigativa, por citar algunas: i) Las originadas por la fusión de las teorías enunciadas en los trabajos de Forrester y del premio Nobel de Economía Simón (1978), las cuales se apoyan en las técnicas propias de la ingeniería de control para analizar el comportamiento de la gestión del inventario [9]; ii) Las basadas en la simulación de sistemas, entre las que destaca el "Juego de la Cerveza", método ideado por Sterman, con el cual muestra de una forma sencilla la creación del efecto como una consecuencia de una inapropiada sincronización de la información de corto plazo (Aproximadamente un horizonte de planificación de doce semanas) en la CdS por parte de sus agentes [10].

\subsection{DEFINICIÓN DEL EFECTO LÁTIGO.}

El Efecto Látigo es un fenómeno que dificulta la gestión administrativa tanto al in- terior como al exterior de las CdS [5-11] y que consiste en una distorsión creciente de la demanda transmitida por los distintos agentes participantes en la gestión del flujo de productos a medida que nos alejamos del mercado [5]. En otras palabras, el efecto Bullwhip, refleja el aumento de la incertidumbre a medida que se transmiten los pedidos aguas arriba en la $C d S$, en tal sentido, es considerado este efecto como el fenómeno de "amplificación" de la deman$\mathrm{da}$, conocida entre los diferentes elementos que componen una CdS en particular [5].

Los motivadores que dan origen a la aparición del Bullwhip al interior de una CdS en particular son [5]: i) Un movimiento oscilatorio de la demanda, cuyas fluctuaciones nada tienen que ver con la original del mercado, dando lugar a una estacionalidad irreal, lo que origina ajustes inadecuados, por ejemplo, en las capacidades de producción o de suministro; ii) Un efecto de amplificación consistente en aumentar las diferencias entre picos y valles, lo que induce falsas expectativas en los agentes; y iii) Un retraso temporal en la información recibida, causante de desfases entre los objetivos reales y la aplicación de políticas tendientes a alcanzarlos, aumentando los índices de incumplimiento.

El predominio de alguna de las tres componentes citadas en el párrafo sobre las demás, dependerá de las condiciones operativas existentes en cada uno de los agentes que integran una misma $C d S$, en el mismo sentido, es de mencionar que el Efecto Látigo es causado por la acción conjunta de estos tres elementos. Al revisar publicaciones 
recientes sobre el tema, sujeto de estudio en esta investigación, éstas giran en torno al estudio de la oscilación de la demanda por ser el principal motivador y el que más impacto genera en la creación del Bullwhip a nivel de la CdS frente a los otros dos componentes, pues cualquier incremento que experimente este componente incide directamente en: i) Los niveles del inventario gestionado en forma conjunta; ii) Las necesidades de recursos requeridos por el área de operaciones; y iii) Demás factores que alteran no solamente la planeación operacional, sino también las proyecciones en edificabilidad en especial, las relacionadas con las naves de almacenamiento de la CdS. La medida matemática del Efecto Látigo en términos de la oscilación de la demanda que experimenta una $C d S$, relaciona a los cuadrados de los coeficientes de variación de la demanda transmitida y recibida, partiendo del supuesto que en el medio y largo plazo los valores promedios de las demandas permanecen constantes (Véase ecuación 1) [5].

$$
B W=\frac{\frac{\operatorname{Var}(q)}{d_{q}^{2}}}{\frac{\operatorname{Var}(d)}{d_{d}^{2}}}=\frac{\operatorname{Var}(q)}{\operatorname{Var}(d)}
$$

En donde: i) $\boldsymbol{B} \boldsymbol{W}$ : Es el indicador empleado para medir el Efecto Látigo producido por un escalón de la cadena; ii) $\operatorname{Var}(q)$ y $\operatorname{Var}(d)$ : Corresponden a las varianzas de ambas demandas, la transmitida $q$ y la recibida $d$, respectivamente, por dicho escalón; y iii) $\boldsymbol{d}_{\mathrm{q}}$ y $\boldsymbol{d}_{\mathrm{d}}$ : Son las demandas medias transmitida $q$, y recibida $d$.

\subsection{REPERCUSIONES DEL EFECTO LÁTIGO EN LAS CADENAS DE SUMINISTRO}

Para Blinder, no existe una regla general que relacione los incrementos que experimentan las órdenes de pedidos cuando hay distorsión en la información inherente a la demanda estimada, es decir un antiBullwhip [1]. Aunque existe la hipótesis que esta afirmación se debe a que Blinder utilizó series cronológicas de tiempo procedentes del Departamento de Comercio de los Estados Unidos poco aptas para el análisis. Por citar un ejemplo, algunas de las series correspondientes a los inventarios estaban elaboradas con las valoraciones contables de las empresas en lugar de unidades físicas, o bien no se contemplaba con exactitud los efectos de la estacionalidad [5].

Otros autores definen la amortiguación de la demanda con el nombre de "Paradoja del Tiempo" [5]. Esta paradoja define el retraso del flujo de información, se ha visto que hay determinadas circunstancias en las que un aumento en estas demoras atenúa la demanda real a lo largo de la CdS, aquí intervienen múltiples factores que muchas veces son estrategias que usan los diferentes agentes de la cadena para optimizar su operación, en tal caso, algunos proveedores de la cadena retrasan los pedidos a sus compradores con el fin de aumentar el tamaño de las órdenes y así incurrir en menores costos de despacho y transporte lo cual, incrementa la distorsión de la demanda. Por otra parte, los retrasos de las órdenes en curso, permiten acumular mayores nive- 
les de información sobre la demanda y, por ende, mejorar las estimaciones, con lo que se consigue mitigar el nivel de incertidumbre que experimenta la demanda transmitida.

Al analizar estas dos situaciones, se puede considerar que existe un punto medio donde el retraso de información no genera una gran distorsión de la demanda, si se une a esta disparidad lo complicado que es el entrelazar los datos existentes entre los diferentes miembros de la cadena, ya que los datos de estos miembros son recogidos con objetivos distintos y en intervalos de tiempos diferentes, hace que muchas veces lo que sea importante para uno es superfluo para otro; al revisar estos elementos y cómo se conjugan, permite concluir que el lograr definir la importancia y el alcance del Bullwhip es complejo, no hay una verdad única sobre cómo enfrentarlo, esto muestra que las repercusiones que sufren las CdS no son diagnosticables enteramente y de forma infalible, las situaciones acontecidas en cada eslabón dependen mucho de las coyunturas del mercado y estrategias específicas que se estén presentando en ese espacio de tiempo (Paros, escases, sobreoferta, tratados, entre otros factores) [5-12].

\subsection{CAUSAS DEL EFECTO LÁTIGO}

El Bullwhip surge a consecuencia de las fluctuaciones en los pedidos que alteran y modifican la información de la demanda dentro de la CdS, las cuales proceden de los siguientes focos: i) Condiciones del merca- do; y ii) Provenientes de la actuación de los agentes que componen la CdS. Cuando las distorsiones se originan por condiciones de mercado, están haciendo referencia a la capacidad de respuesta que poseen los agentes logísticos encargados del flujo de materiales al interior de la CdS, para atender cambios inesperados en la demanda, que superen los niveles permisibles de la UCE del sistema logístico en general [1314]. Con respecto a la forma de actuar de los actores logísticos se está haciendo referencia a las causales más comunes que las originan, a saber: i) Las provocadas por las ineficiencias en la gestión operacional de la CdS (Por citar algunos ejemplos: i) Tiempos de respuesta subestimados; ii) Elevados costos logísticos; iii) Métricas desfasadas en los SKU, entre otros aspectos); y ii) Las relacionadas con situaciones exógenas a los procesos de planificación conjunta de la CdS (Por ejemplo: i) Procesos especulativos en la coordinación de la UCE; ii) Renuncia a desarrollar procesos de mejora continua sostenibles, entre otros aspectos). En términos generales, todo proceso colaborativo es de naturaleza compleja, motivo por el cual, la presencia de oscilaciones es inherente al propio sistema [13-14].

Según el investigador Hau Lee, considera que las causas que dan origen a las perturbaciones que pueden experimentar una $\mathrm{CdS}$ son [11-12]: i) La utilización indiscriminada de los métodos de predicción por parte de sus agentes; ii) Tamaño de las órdenes cursadas no gestionadas de forma conjunta por la dupla comprador-vendedor; iii) Alteraciones no planificadas de los precios de 
mercado; y iv) El uso de esquemas de negociación especulativos y/o de sobreprotección de la UCE. Así mismo, Helbing cita las siguientes cinco condiciones que permiten auto-regular en forma eficiente las posibles variaciones generadoras del efecto Bullwhip al interior de una CdS en particular [13]: i) La estimación de la demanda realizada por los agentes deberá contemplar un comportamiento estacionario y transferida hacia el mercado; ii) Tiempos de respuesta pactados entre los agentes; iii) No poseer agotados en cada nodo; iv) Ausencia de costos fijos en la emisión de órdenes de reposición; y v) Precio de mercado estacionario del producto.

Los autores de la presente investigación coinciden totalmente con lo expuesto por Helbing y Hau Lee en el párrafo anterior, y a su vez, armonizan con el Dr. Martín-Andino en torno a la inclusión de causales que dan origen al Efecto Látigo, debido a las condiciones actuales del mercado nacional [6-7], estas causales son divididas por él en cuatro grupos, a saber [5]: i) Perturbaciones debidas a sesgos introducidos en la demanda. Demanda de mercado no estacionaria. Bien sea no estacionaria en demanda o en varianza o en co-varianza. Modificación del modelo matemático de predicción o en los parámetros utilizados en el mismo modelo. Cambios en la cadencia del muestreo de la demanda. Cambios en los precios de venta por el agente cliente (A modo de ejemplo, promociones, descuentos por cantidad, cheques regalo, bonos de compra, entre otras estrategias de marketing). Perturbaciones introducidas por los tiempos de repuesta; ii) Cambios en los tiempos de tratamiento de la información. Cambios en los tiempos de suministro. Tiempos de suministro estocásticos; iii) Prácticas logísticas inapropiadas. Tamaño variable de las órdenes de reaprovisionamiento supuesto que la elasticidad de la demanda respecto al precio propio no sea infinita; esto es, un mercado en el que cambios en los precios originan cambios en la demanda. Desde este punto de vista y por concretar más este aspecto, se diría que podría darse el caso de haber interferencias en la demanda de un producto provocadas por los precios de otros, hace referencia a productos complementarios o sustitutivos; en estas circunstancias también se crearía una perturbación de la demanda. Cambios en los niveles de cobertura de los inventarios (Por ejemplo, reacciones imprevistas de los agentes en busca de sobreprotección); y iv) Alteración de las condiciones de la red de distribución. Cambios en los precios de ciertas actividades logísticas (Por ejemplo, transportes). Cambios en el número de agentes clientes dependientes de un escalón. Modificaciones en la topología de la red.

\section{MÉTODOS DE ATENUACIÓN Y MEDICIÓN DEL EFECTO LÁTIGO (BULLWHIP) EN LA CADENA DE ABASTECIMIENTO.}

\subsection{MODELO DEGESTIÓN DE INVENTARIOS Y POLÍTICA DE REPOSICIONES OR- DER UP TO LEVEL}

En el modelo de gestión OUT, los pedidos colocados por un cliente a su proveedor quedan determinados por el modelo de Forrester (Véase ecuación 2) [8]. 


$$
\left\{\begin{array}{l}
\text { Órdenes Cursadas } \\
\text { al Proveedor en } t+1
\end{array}\right\} \quad\left\{\begin{array}{l}
\text { Demanda del Cliente } \\
\text { en Periodo } t
\end{array}\right\} \quad\left\{\begin{array}{l}
\text { Diferencias entre el inventario objetivo } \\
\text { estimado en ty el inventario objetivo } \\
\text { estimado en } t-1
\end{array}\right\}
$$

La meta perseguida por esta política de gestión del inventario es minimizar los costos de almacenamiento y de déficit (Ventas no realizadas por agotados). Ante una variación de la media o varianza en la demanda (Situación que es percibida por el responsable del bodegaje de producto como una inestabilidad creciente en el sistema de inventarios) es conveniente cambiar de política de gestión de órdenes de reposición, donde la recuperación del inventario sea gradual, con el objeto de mitigar el efecto Bullwhip. Esta política es una variación de la cita anterior, donde por inventario disponible se toma el inventario físico más el que se encuentra por recibir o en proceso de fabricación, tomando a $K_{1}$ como un factor comprendido entre 0 y 1 cuyo sentido físico es asimilable a una constante de recuperación del inventario y que determina la estabilidad de la cadena siendo 0 cuando la cadena es inestable y 1 cuando muestra estabilidad (Véase ecuación 3) [15].

$C_{t}=\hat{d}_{t}+K_{l}\left(I D_{0}-I D_{t}\right)=\hat{d}_{t}+K_{l}\left(I_{0}-I_{t}\right)+K_{l}\left(P_{0}-P_{t}\right)$

En dónde: i) $C_{t}$ es la orden cursada al proveedor; ii) $\hat{d}_{t}$ es la estimación de la demanda para el periodo próximo $t$; iii) $D_{0}$ es el inventario disponible objetivo para el periodo $t_{i}$ y iv) $D_{t}$ es el inventario disponible en el periodo $t-1$. Con respecto al inventario físico objetivo $I_{0}$ éste viene expresado como la suma entre el inventario de consumo anual y el inventario de estabilidad del sistema (Véase ecuación 4) [5].

$$
I_{0}=D_{l}+K \sigma_{\varepsilon, L}
$$

Dónde: i) ${ }_{\varepsilon, L}^{\sigma}$ es la desviación estándar de los errores del pronóstico durante el periodo $L$ (Representa el tiempo transcurrido (TS) desde que el agente cursa un pedido hasta que se recibe y está disponible en su almacén, más el periodo de revisión $T R$ o tiempo transcurrido entre pedido y pedido). Se toma TR con valor de 1 periodo y TS se expresará en múltiplos de TR (Véase ecuación 5) [5].

$$
L=T S+1
$$

Por su parte, $D_{L}$ es la estimación de la demanda para el periodo $L$, y $K$, es el factor de seguridad, por consiguiente, el inventario objetivo de órdenes pendientes es el resultado de multiplicar la demanda promedio por el tiempo de respuesta pactado para el producto en consideración, siendo $\bar{d}$ la demanda media (Véase ecuación 6) [5].

$$
P_{0}=\bar{d} * L
$$

Para finalizar, el algoritmo a utilizar en los procesos de reposición del inventario sería: i) Al inicio del periodo $t$ se recibe el pedido 
cursado durante $L$ periodos anteriores; ii) Se recibe la orden de compra teniendo claro el $d_{t}$ del producto; iii) El proveedor recibe la orden de compra y revisa sus niveles de existencias, y deberá a su vez proyectar la demanda para el periodo $L+1$; iv) El proveedor debe consolidar todas sus órdenes de pedido en firme (Demanda real) para el $L+1$, esto teniendo en cuenta la diferencia dada entre la resta de su nivel de inventario mínimo y el actual, sumándole también las unidades por ingresar o que están en producción, más los pedidos ingresados; y v) El proveedor debe montar los pedidos antes de terminar el periodo $t$ aplicando la ecuación 3, y definiendo a $K_{L}=K_{o}+K_{p}$ aplicando distintos valores constantes $K_{o}$ para el inventario físico y $K_{p}$ para el inventario de órdenes pendientes. Así se puede adaptar mejor una respuesta por parte del agente frente a las fluctuaciones de la demanda de mercado.

\subsection{EOQ CANTIDAD ECONÓMICA DE PEDIDO}

Para facilitar el flujo de información al interior de la CdS, una posible medida que se puede adaptar gira en torno a la cuantificación de la UCE de costo razonable [16]. En el caso concreto de esta investigación se ha considerado que el modelo EOQ es funcional para la CdS de blanqueadores (Véase ecuación 7) [15-16].

$$
E O Q=\sqrt{\frac{2 * C_{0} * D}{P * i}}
$$

En dónde: i) Co representa el costo de pedir (Los cuales pueden ser resumidos en gastos de administración y comunicaciones); ii) $D$ es la demanda requerida en un periodo $L$ de tiempo (Expresada en términos de t, Its, $\mathrm{Kg}$ entre otras por unidad de L); iii) $P$ es el costo unitario de la compra (El cual encierra impuestos, fletes, costos de compra, seguros y embalajes); y iv) i constituye la tasa de transferencia (Incluye los costos de financieros y de bodegaje).

\subsection{ANÁLISIS DEL BULLWHIP EFFECT EN LA CdS DE BLANQUEADORES}

Normalmente el Efecto Látigo y sus consecuencias se miden utilizando la ecuación que viene definida como el cociente de la varianza entre la demanda transmitida $\sigma_{q}^{2}$ y la demanda recibida $\sigma_{d}^{2}$ (Véase ecuación 1) [5]. Sin embargo, el Efecto Látigo es más que una amplificación de la demanda, ya que altera su frecuencia, afecta la arquitectura empresarial requerida por la $\mathrm{CdS}$ en términos de tamaño y velocidad a la cual deben ir los datos, aumentando el diferencial de demanda entre los periodos de planificación picos y valles retrasando la recepción de los pedidos conjuntos, haciendo que la varianza sea = 1, permitiendo una fuerte distorsión de la demanda, debido a una estacionalidad diferencial entre las órdenes cursadas al proveedor y la demanda recibida por los clientes [5-12].

En ningún caso el cociente de varianzas diferencia entre las diversas causas que originan el Efecto Látigo [5-11], como sí sucedería si los tamaños de los lotes solicitados al proveedor son muy distintos al 
de los recibidos de sus clientes [5-11-12]. Para el caso que nos concierne el índice de Efecto Látigo calculado para la CdS de blanqueadores está alrededor del 0.9025, este valor se puede interpretar como el grado de distorsión de la demanda recibida por la compañía líder en la línea de blanqueadores y la demanda despachada aguas arriba, que comparado contra la regla del $85 \%$ de planificación de sistemas productivos se puede concluir que, esta compañía posee un desequilibrio estacional entre las órdenes cursadas al área de operaciones y la demanda enviada a sus clientes (Equivalente a 1).

\section{ANÁLISIS DE LOS COMPONENTES DE ESTACIONALIDAD Y TENDENCIA DE LA DEMANDA}

El análisis estadístico de la demanda, busca establecer el comportamiento del mercado meta de la firma en un horizonte de planeación táctica (Últimas sesenta semanas de negociación) y poder tener un conocimiento del nivel de variabilidad que proyecta el modelo matemático seleccionado en términos del Error Cuadrático Medio (ECM) [17-18]. Así mismo, el planificador del sistema logístico puede establecer que tan agresivos son los cambios generados en la demanda proyectada una vez se ajuste a las condiciones del sector económico al que pertenece la CdS en un formato de descomposición de series de tiempo [19].

\subsection{ANÁLISIS DE LA TENDENCIA}

Movimiento persistente de la demanda a través del tiempo. Se presenta por cam- bios en la tecnología, avances científicos, incrementos en la población, entre otros aspectos. Para identificar la existencia o no de la tendencia en una serie cronológica de tiempo, se realiza la prueba de Anova con respecto al periodo de tiempo de análisis (En los sistemas ERP la unidad básica de tiempo es la semana) para determinar si los promedios de la demanda estimada para el horizonte de planeación táctica presentan o no diferencias significativas [20]. Para casos prácticos, el nivel de significancia recomendado por los especialistas en análisis de datos se cifra en 0.05 para pruebas de Anova. Con respecto al análisis hecho a los datos experimentales de la presente investigación esta prueba determinó que al menos uno de los promedios de la demanda es diferente ( $p=0.000<0.05)$, confirmando que la serie cronológica de tiempo presenta tendencia.

\subsection{ANÁLISIS DE LA ESTACIONALIDAD}

Son fluctuaciones periódicas regulares que se presentan en las series cronológicas relacionadas con las ventas de la CdS cada doce meses, con repetición anual. Estas se originan por condiciones climatológicas, costumbres sociales y religiosas entre otras razones [18-19]. Para identificar la existencia o no del componente estacional en una serie cronológica, se realiza la prueba de Anova con respecto a los periodos mensuales de ventas, siguiendo los mismos lineamientos dados en el apartado anterior para el análisis de tendencia [20]. Con respecto al análisis realizado a los datos históricos de demanda, entregados por la compañía 
base de esta investigación, no se evidenció al nivel de significancia del 0.05 la presencia del componente estacionario en dicha serie cronológica.

\section{INFLUENCIA DEL MÉTODO DE PREDICCIÓN DE LA DEMANDA EN EL MODELO BULLWHIP EFFECT}

Dependiendo del método de pronóstico usado para estimar la demanda se puede establecer cómo se comportan los agentes que integran la CdS aguas arriba y qué tanto la estabilizan [5-11].

\subsection{ALISADO EXPONENCIAL SIMPLE}

El modelo de Alisado Exponencial Simple (AES), posee la virtud de ser sencillo de utilizar, siendo el dato proyectado para el siguiente período una expresión simple del valor real del período anterior y de la previsión realizada para ese mismo período [18-19]. Sin embargo, resulta de vital importancia seguir las siguientes pautas de desarrollo de modelado [17-18-19]: i) Para iniciar la secuencia de previsión se requiere un dato semilla (Para ello, el proyectista suele utilizar uno de los siguientes procedimientos: i) Toma el primer dato real de la serie cronológica; ii) Usa un promedio con respecto a los 2 o 3 primeros datos de la serie histórica de demanda; y iii) Un valor de previsión obtenido por otros procedimientos); $y$ ii) La técnica tiene memoria limitada a un período de previsión (Puede evidenciarse con facilidad esta característica inhe- rente al modelo matemático que, en ausencia de realizaciones de la serie cronológica, al intentar prolongar la previsión más allá de un período, provoca que las proyecciones sean sucesivamente iguales).

La formulación matemática del modelo AES se origina en la función transformada $Z$ de parámetro $\alpha$ (Véase ecuación 8) [5].

$$
\mathscr{f}(Z)=\propto f(Z)=\frac{\alpha^{*} z}{z-(1-\alpha)}
$$

La ecuación 8 se incorpora en la proyección del inventario $L$ períodos futuros de planificación táctica (Véase ecuación 9) [5].

$$
I_{0}(z)=L^{*} F(z)+C
$$

En donde la constante $C$ representa el inventario de seguridad (El cual no cambia en el tiempo como consecuencia que los factores que lo determinan no están en función del momento en que se cuantifica). Seguidamente, se deberá cuantificar el inventario objetivo en función de la transformada $Z$ (Véase ecuación 10) [5].

$$
\vartheta_{0}=L \frac{\alpha * Z}{Z-(1-\alpha)}
$$

Se remplaza $L$ por $K_{L^{\prime}}$ para indicar que se va a ensayar este parámetro con diversos valores durante el proceso de experimentación del modelo AES, este procedimiento afecta la cuantificación del inventario objetivo de órdenes en curso (Véase ecuación 11) [5]. 


$$
P_{0}=\bar{d} * K_{L}
$$

La proyección de la demanda se realizará por medio de la aplicación de un promedio móvil simple de orden cinco, con el objeto

$$
\bar{d}=\frac{X_{t}+X_{t-1}+X_{t-2}+X_{t-3}+X_{t-4}}{5} \quad \rho_{0}(z)=K_{L} \frac{1+Z+Z^{2}+Z^{3}+Z^{4}}{5 Z^{4}}
$$

Finalmente, se sustituyen los diferentes modelados en la función de transferencia genérica (Véase ecuación 13). Con rela- de transferir a todos los actores miembros de la CdS las variaciones que puede experimentar sin ningún efecto amortiguador (Véase ecuación 12) [5].

ción a los parámetros $K_{\mu} K_{p}$ y $K_{L}$ han de ser ajustados para cada valor de $\alpha$ simulado durante el proceso de experimentación [5].

$$
\frac{C(z)}{D(z)}=z^{L-1} \frac{(z-1)\left[\left(1+K_{I} L \frac{\alpha * Z}{z-(1-\alpha)}+K_{P} K_{L} \frac{1+Z+Z^{2}+Z^{3}+Z^{4}}{5 Z^{4}}\right]+K_{I} z\right.}{z^{L}-\left(1-K_{P}\right) z^{L-1}+K_{I}-K_{P}}
$$

La Figura 1, muestra el mejor escenario de estimación de las órdenes solicitadas al proveedor en términos de la UCE de blanqueadores, modelado con la técnica de predicción AES [21]. Para analizar la respuesta arrojada por el sistema de proyección en estado estable, los investigadores consideraron los siguientes indicadores: i) Pico máximo alcanzado por la serie "Pedidos al Proveedor de la UCE de blanqueadores" sobre la media de la serie "Demanda Ajustada de la UCE de blanqueadores" una vez transcurrido el estado transitorio, $51.60 \%$; ii) Tiempo de recuperación del inventario físico, 0.34 días; iii) ECM de la proyección a un período, 358,887 UCE de blanqueadores; y iv) ECM como diferencia entre "Pedidos al Proveedor" y "Demanda Ajustada", 72.764 UCE de blanqueadores.

Del análisis realizado al indicador del Efecto Látigo (BW) medido como cociente de las varianzas de la serie "Pedidos al Proveedor" y "Demanda Ajustada", calculado a partir de la observación 72, momento en el que se considera que el sistema está en estado estable (BW = 1), no se evidencia que existe Efecto Látigo ni efecto anti-látigo. En todo caso, a medida que la demora aumenta, las respuestas desmejoran y se hacen más sensibles a los cambios de los parámetros de estimación. 


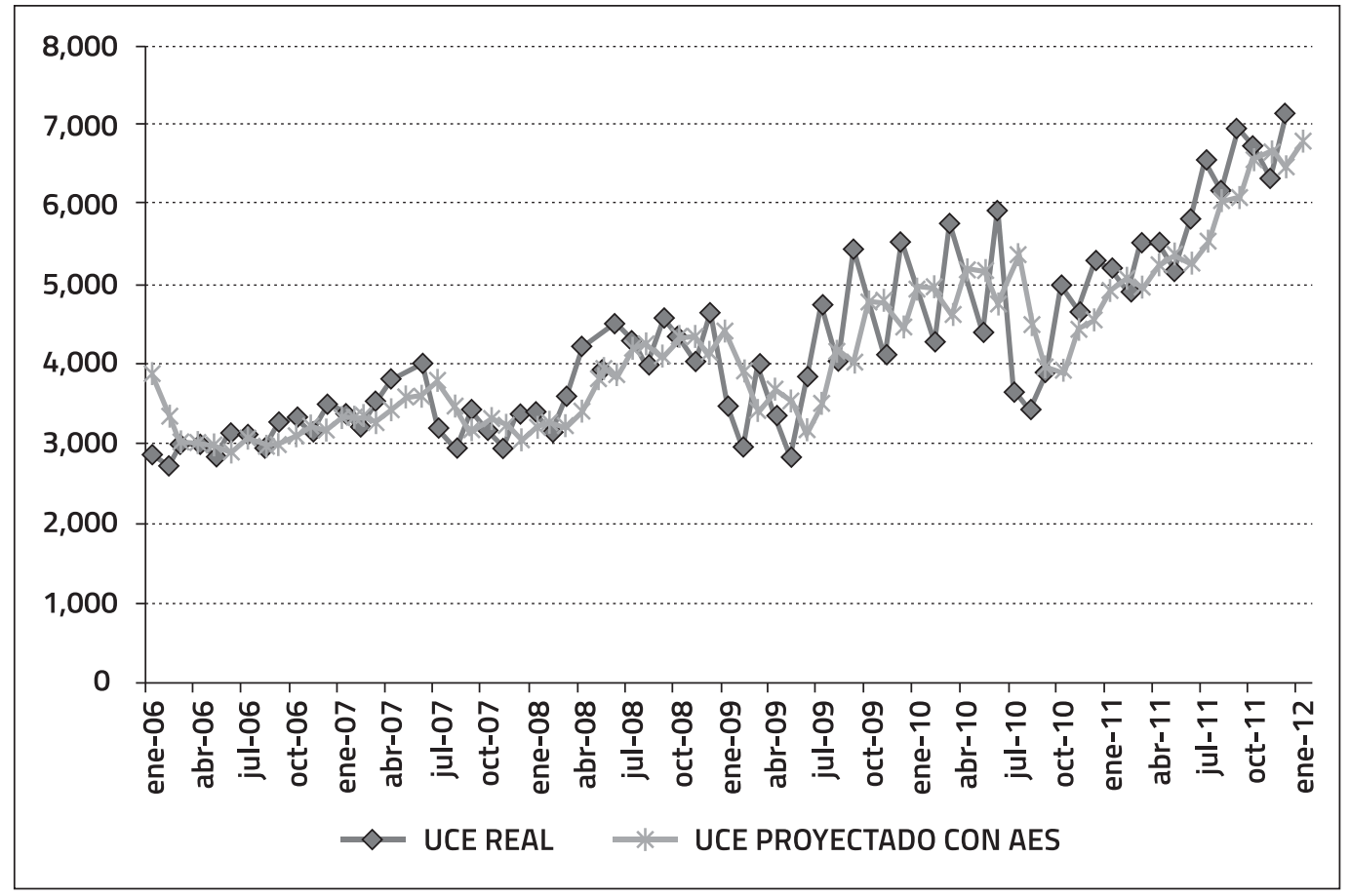

Figura 1. Representación Gráfica de la Demanda Proyectada con el modelo AES

Fuente: Autores

\subsection{ALIZADO EXPONENCIAL HOLT -WINTERS}

Esta técnica de predicción incorpora en el modelado al componente de tendencia que trae la serie histórica de datos, superando así las restricciones que poseen los modelos de Medias Móviles como también el modelo AES [18]. Por tanto, este método es aconsejable cuando la serie cronológica muestre una cierta tendencia a crecer o decrecer [17]. El modelado matemático de Holt-Winters consta de tres fases de análisis: i) El nivel; ii) La tendencia; y iii) La estacionalidad. Estas tres fases de análisis están relacionadas entre sí por ecuaciones iterativas, que integran los parámetros de simulación de nivel, tendencia y estacionalidad que oscilan entre 0 y 1 , que a su vez son denotados como $\alpha, \beta$ y $\lambda$ respectivamente [19].

La interpretación que se le puede dar a los parámetros desde el punto de vista cuantitativo es la siguiente [17-18-19]: i) $\mathrm{Si} \beta=\lambda$ $=0$, el modelado se reduce a una expresión matemática y el resultado de la predicción se reduce a un proceso de $\mathbf{A E S}$; $y$ ii) $\operatorname{Si} \lambda=$ 0 , el modelado se reduce a un proceso de alisado exponencial de Holt, el cual no tiene en cuenta el componente estacional. Para usar este método, es necesario estimar el valor de la serie suavizada $\left(e_{i}\right)$ y el valor de la tendencia $\left(T_{i}\right)$ durante todo el proceso de 
modelado de la serie cronológica (Véase ecuación 14) [19].

$$
\begin{aligned}
& e_{i}=U\left(e_{i-1}+T_{i-1}\right)+(1+U) Y_{i} \\
& T_{i}=V T_{i-1}+(1-V)\left(e_{i}-e_{i-1}\right)
\end{aligned}
$$

En donde: i) $e_{i}$ Representa el nivel de la serie suavizada en el período i; ii) $T_{i}$ constituye el valor del componente de tendencia para el período i; iii) $Y_{i}$ es el valor observado de la serie cronológica en el período $i ;$ y iv) Las expresiones $\mathrm{U}$ y $\mathrm{V}$ son constantes de suavización.

La Figura 2, muestra el mejor escenario de estimación de las órdenes solicitadas al proveedor en términos de la UCE de blanqueadores, modelado con la técnica de predicción Holt-Winters [18]. Para analizar la respuesta arrojada por el sistema de proyección en estado estable, los investigadores consideraron los siguientes indicadores: i) Los parámetros principales del modelo Holt-Winters son: $\alpha=0.1500 ; \beta=0.8500$; y $\lambda=0.0000$; ii) Base del Pronóstico $\left(A_{0}\right)=$ 3.875 UCE de blanqueadores; iii) Tendencia Base Decreciente $\left(T_{0}\right)=5.16$ UCE de blanqueador; iv) Pico máximo alcanzado por la serie "Pedidos al Proveedor de la UCE de blanqueadores" sobre la media de la serie "Demanda Ajustada de la UCE de blanqueadores" una vez transcurrido el estado transitorio, 15\%; v) Tiempo de recuperación del inventario físico, 0.34 días; iii) ECM de la proyección a un periodo, 455,067 UCE de blanqueadores; y iv) ECM como diferencia entre "Pedidos al Proveedor" y "Demanda Ajustada", 86,839 UCE de blanqueadores.
Del análisis realizado al indicador del efecto látigo (BW) medido como cociente de las varianzas de la serie "Pedidos al Proveedor" y "Demanda Ajustada", calculado a partir de la observación 72, momento en el que se considera que el sistema está en estado estable (BW $=1)$, se evidencia que existe Efecto Látigo (BW Calculado $=0.8762)$. En todo caso, al proyectar la demanda de la UCE de blanqueadores con la técnica de Holt-Winters el volumen de ventas proyectadas se ve disminuido en un $2.7 \%$ anual con respecto a la proyección realizada con la técnica AES.

\section{CONCLUSIONES}

El Efecto Látigo que administra el agente que posee el poder de negociación (Firma de referencia para esta investigación) en la CdS de blanqueadores, es debido a las variaciones no planificadas de la demanda provenientes del mercado (Los miembros de este sistema logístico tiene por costumbre inflar los pedidos para no presentar rupturas de ventas) que se refleja como una deficiencia en la métrica de la UCE, situación que genera un desequilibrio entre la demanda recibida por la compañía líder y la demanda despachada aguas arriba en el mediano plazo $\left(B W_{\text {Calculado }}=0.9025\right)$. Es de mencionar, que el grado de afectación que pueda poseer una CdS con relación al Wullhwip Effect varía entre 0 y 1 , en donde 1 es que presenta total perturbación y en 0 en caso contrario, de ahí que se pueda afirmar que la compañía estudiada presenta una afectación severa, es decir, que los datos planificados para la demanda distan de la realidad. 


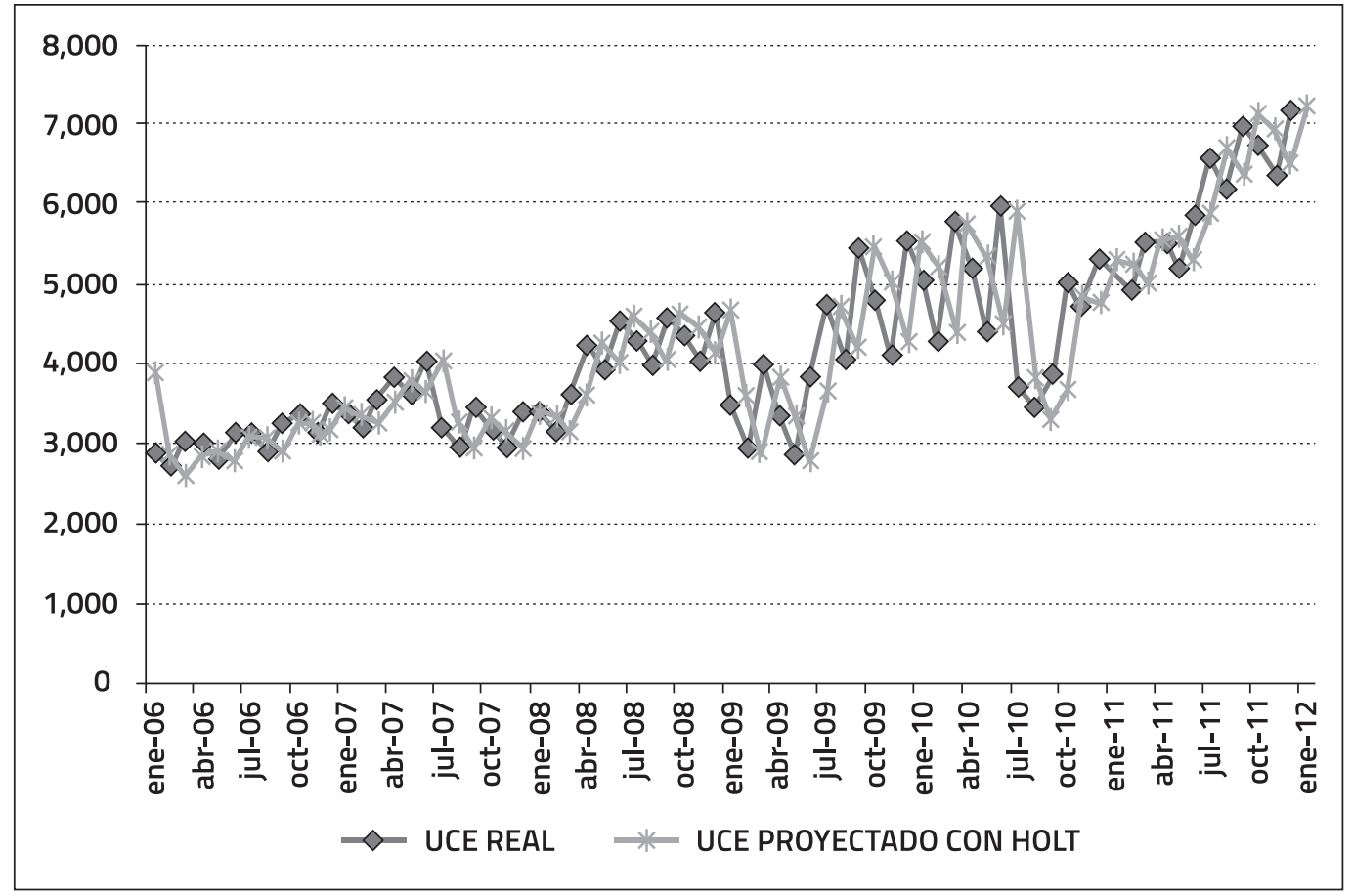

Figura 2. Representación Gráfica de la Demanda con el Modelo Método Holt-Winters.

Fuente: Autores

La UCE de blanqueadores que controla el Efecto Látigo se cuantificó en función del modelo de coordinación EOQ con: i) Costo conjunto, $\mathrm{CT}_{\text {AGENTE LIDER }}=1,075^{*} \mathrm{CT}_{\text {CdS AGUAS }}$ arRisa' $y$ ii) SKU de Negociación (Mínimo), Decena de cajas de 24 Unidades de 500 CC. En general, la línea de blanqueadores está configurada con productos de bajo margen de utilidad, razón por la cual, los autores de la presente investigación sugirieron el empleo de esta UCE a la CdS de blanqueadores que les permitirá racionalizar los sobrecostos evitando pérdidas excesivas en la rentabilidad conjunta.

La CdS de blanqueadores requiere revisar su: i) Modelo de negocio; ii) Arquitectura
Empresarial; y iii) Planeación Estratégica, con el objeto de estabilizar en el largo plazo sus procesos de: i) Integración; ii) Sincronización; y iii) Coordinación, que les permitan mitigar el Efecto Látigo de una forma más planificada y coherente con los principios universales de sostenibilidad.

La proyección de la demanda con la técnica AES permite mitigar el Efecto Látigo de la CdS de blanqueadores, con un manejo de la UCE de 26 decenas por despacho (6.240 unidades) con una rotación aproximada de 782 pedidos anuales, situación que le representa al agente que posee el poder de negociación en esta cadena, una capacidad de producción y manipulación logística del 
orden de las 3.250 UCE por mes en promedio, que son manufacturadas en tres turnos de trabajo por día.

Para finalizar, los autores de la presente investigación no pretenden generalizar el resultado obtenido por ser éste un caso particular cuando las cadenas sufren un desfase temporal en su demanda en algún punto de su flujo. Por otra parte, el Efecto Látigo en términos generales incide en la planeación operacional, motivo por el cual no se justifica suponer que a mediano y largo plazo los promedios de las demandas (Transmitidas y recibidas) se mantengan y por el contrario, sí lo hagan sus varianzas. Esto significa, que el modelado matemático no trasgrede para nada la problemática que pueda poseer un sistema logístico en particular frente a la generación del Efecto Látigo.

\section{REFERENCIAS}

[1] Blinder, A.S., (1999). El Banco Central: Teoría y Práctica. Barcelona. Antoni Bosch Editor, $112 \mathrm{p}$.

[2] Campuzano Bolarin, F., et al, (2008). Consecuencias del Efecto Bullwhip según distintas estrategias de gestión de la Cadena de Suministro: Modelado y Simulación. En: Revista de Métodos Cuantitativos para la Economía y la Empresa, Vol. 5, pp. 49-66.

[3] Ros Mcdonnell, L., et al, (2006). Variabilidad de la demanda y sus efectos según distintas políticas de gestión de la Cadena de Suministro. Modelado y Simulación. En: http://www.adingor.es/Documenta-
ción/ClO/cio2006/docs/000082_final. pdf (Febrero del 2012).

[4] Disney, S.M., and Towill, D.R., (2003). The Effect of Vendor Managed Inventory (VMI) Dynamics on the Bullwhip Effect in Supply Chain. En: International Journal of Production Economics, Vol. 85, Issues 2, pp. 199-215.

[5] Martín-Andino Benítez R.J., (2006). El Efecto Látigo (Bullwhip) En las Cadenas de Suministro y la Dependencia de los Agentes que las Integran. Tesis Doctoral. Facultad de Ingeniería. Departamento de Organización Industrial. Universidad Pontificia de Comillas de Madrid. Madrid, 319 p.

[6] Departamento Administrativo Nacional de Estadística; (2002). Encuesta Anual Manufacturera de 2002. Bogotá D.C., CPC.

[7] MUNDOBIZ S.A.S; (2011). Vademécum Empresarial 2009/2010: Grandes sectores: Químicos y Agroquímicos (Producción y Comercialización). En: Http://www.lanotadigital.com/vademecum/big/quimicos/ laboratorios-farmaceuticos (Octubre del 2011).

[8] Forrester, J.W., (1961). Industrial Dynamics. $2^{\text {th }}$ ediction. MIT Press, $464 \mathrm{p}$.

[9] Simón, Herbert A., (1978). Rational Decision-Making in Business Organizations. Nobel Memorial Lectura 8, En: The American Economic Review, Vol. 69, pp. 493-513. 
[10] Sterman, John D., (1989). Modeling Managerial Behavior: Misperceptions of Feedback in a Dynamic Decision Making Experiments. En: Managent Science, Vol. 35, Issue 3, pp. 321-339.

[11] Lee, Hau L., et al, (1997). The Bullwhip Effect in Supply Chains. En: MIT Sloan Management Review, Vol. 38, pp. 93102.

[12] Lee, Hau L., et al, (1997). Information Distortion in Supply Chain: The Bullwhip Effect. En: Management Science, Vol. 43, Issue 4, pp. 546-558.

[13] Helbing, D., et al, (2004). Physics, stability and dynamics of supply network. En: Physical Review Dresden, University of Technology, Vol. 70, Issues 6, pp. 1-6.

[14] Mason-Jone, R, et al, (1997). The impact of pipeline control on supply chain dynamics. En: International Journal of Logistics Management, Volume 8, Issue 2, pp. 4762.

[15] Silver, E.A., and Peterson, R., (1985). Decision systems for inventory management and production planning. $2^{\text {th }}$ edition, John Wiley and Sons, 722 p.
[16] Porteus, Evan L., (1985). Investing in reduced setups in the EOQ model. En: Management Science, Vol. 31, Issue 8, pp. 998-1010.

[17] Sarabia Alegría, J.M., y Pascual Sáez, M., (2009). Curso básico de estadística para Economía y Administración de Empresas. Servicio de publicaciones de la Universidad de Cantabria, $378 \mathrm{p}$.

[18] De la Fuente, D., et al, (1995). Métodos Cuantitativos de Previsión. Servicio de publicaciones de la Universidad de Oviedo, $62 \mathrm{p}$.

[19] González Casimiro, M.P., (2009). Técnicas de Predicción Económica. Serie de documentos Sarriko-on, Universidad del País Vasco (UPV-EHU), $145 \mathrm{p}$.

[20] Díaz Cadavid, A., (1999). Diseño Estadístico de Experimentos. Editorial Universidad de Antioquia, $284 \mathrm{p}$.

[21] BLOGGER-ECONOMÍA EXCEL; (2006). Modelos y aplicaciones Excel para la economía y la gestión de empresas: Alisado exponencial simple. En: Http://www.economiaexcel.com/2010/04/ (Febrero del 2012). 